\title{
EFEITO DA SUPLEMENTAÇÃO COM ACEROLA NOS NÍVEIS SANGÜÍNEOS DE VITAMINA C E DE HEMOGLOBINA EM CRIANÇAS PRÉ-ESCOLARES
}

\section{SUPPLEMENTATION WITH WEST INDIAN CHERRY AND ITS EFFECTS ON THE BLOOD LEVELS OF VITAMIN C AND HEMOGLOBIN IN PRESCHOOL CHILDREN}

\author{
Maria José de Carvalho COSTA' \\ Aline Lacerda de Queiroga TERTO² \\ Leonor Maria Pacheco SANTOS 3 \\ Maria Amélia Amado RIVERA ${ }^{4}$ \\ Luiza Sônia Ascciuti MOURA'
}

\begin{abstract}
RESUMO
O estudo teve como objetivo avaliar a prevalência da deficiência de vitamina C em 104 crianças de 2 a 6 anos de idade de creches municipais de João Pessoa, PB, bem como analisar a eficácia da suplementação com acerola. Foram realizadas dosagens de vitamina $C$ sérica e de hemoglobina antes e depois da intervenção. Os resultados iniciais mostraram prevalência de 69,2\% de carência de vitamina $C(<0,80 \mathrm{mg} / \mathrm{dL})$ e 35,7\% de crianças com níveis de hemoglobina indicativos de anemia ( $\mathrm{Hb}$ $<11,0 \mathrm{mg} / \mathrm{dL}$ ); encontrou-se associação entre os níveis de vitamina $C$ e de hemoglobina $(p<0,01)$. Para as 72 crianças com níveis baixos de vitamina C foi oferecido durante 35 dias, $150 \mathrm{~mL}$ de suco de acerola (teor médio $565 \mathrm{mg}$ de ácido ascórbico/100 mL). A comparação de níveis séricos médios de vitamina $C$ antes e após a suplementação $(0,52+0,20 \mathrm{mg} / \mathrm{dL}$ e 1,43+0,43 $\mathrm{mg} / \mathrm{dL}$, respectivamente) mostrou diferença significativa $(p<0,001)$. Também ocorreu aumento significativo na concentração média de hemoglobina, que de 11,17+1,46 g/dL passou a 12,22 + 1,52 $\mathrm{g} / \mathrm{dL}(p<0,001)$. Considerando-se os resultados obtidos, a disponibilidade e o baixo custo deste fruto regionalmente, justifica-se a proposta de inclusão do mesmo em programas de alimentação para populações de alto risco para a anemia.
\end{abstract}

Termos de indexação: acerola, vitamina C, hemoglobina, pré-escolar.

\begin{abstract}
The objective of this study was to evaluate the prevalence of vitamin C deficiency among 104 preschool children, aged 2 to 6 years, enrolled at municipal day-care centers in João Pessoa, PB, Brazil, and to test the efficacy of supplementing their diet with West Indian Cherry. Determinations of serum vitamin $C$ and blood hemoglobin were carried out before and after supplementation. First results showed $69.2 \%$ of prevalence of vitamin C deficiency $(<0.80 \mathrm{mg} / \mathrm{dL})$ and $35.7 \%$ of children with hemoglobin levels indicative of anemia $(\mathrm{Hb}<11.0 \mathrm{mg} / \mathrm{dL})$; these two indicators were found to be associated $(p<0.01)$. Seventy-two children with low levels of vitamin C were offered, during 35 days, $150 \mathrm{~mL}$ of West Indian

\footnotetext{
(1) Departamento de Nutrição, Centro de Ciências da Saúde, Universidade Federal da Paraíba. Campus Universitário, 58000-900 João Pessoa, PB, Brasil. Correspondência para/Correspondence to: M.J.C. COSTA.E-mail: mjccosta@funape.ufpb.br

${ }^{(2)}$ Mestranda em Ciência e Tecnologia de Alimentos, Universidade Federal da Paraíba.

${ }^{(3)}$ Consultora do Departamento de Nutrição, Universidade Federal da Paraíba.

(4) Departamento de Enfermagem, Universidade Estadual da Paraíba.
} 
Cherry juice (mean content $565 \mathrm{mg}$ of ascorbic acid/100 mL). Comparison of vitamin C levels before and after supplementation $(0.52+0.20 \mathrm{mg} / \mathrm{dL}$ and $1.43+0.43 \mathrm{mg} / \mathrm{dL}$ respectively $)$ showed a significant difference $(p<0.001)$. There was also a significant increase on the mean of hemoglobin concentration, which from $11.17+1.46 \mathrm{~g} / \mathrm{dL}$ rose to $12.22+1.52 \mathrm{~g} / \mathrm{dL}$ after the intervention $(p<0.001)$. Considering the results presented here and the availability of the cherries at low cost in the region, this fruit juice should be included in all feeding programs for populations at risk of anemia.

Index terms: acerola, vitamin C, hemoglobin, child, preschool.

\section{INTRODUÇÃO}

As deficiências em micronutrientes, que atingem principalmente as populações de baixo poder aquisitivo, ocorrem sobretudo devido à disponibilidade inadequada de alimentos fontes de vitaminas e minerais na dieta, tanto do ponto de vista quantitativo como qualitativo. As carências de micronutrientes mais estudados mundialmente são as de vitamina $\mathrm{A}$, ferro e iodo, muito embora ainda persistam outras deficiências, a exemplo de vitamina C ou ácido ascórbico (Santos et al., 1989; Organización Mundial...1990). É preciso também levar em conta, do ponto de vista da saúde pública, as conseqüências do efeito sinérgico entre as deficiências múltiplas de micronutrientes. A interferência negativa do aporte inadequado de vitamina $C$ sobre a absorção do ferro de origem vegetal é bem conhecida. A absorção de sais de ferro é muito afetada pelo ácido ascórbico, enquanto que esta vitamina tem um efeito negligenciavel sobre a absorção de ferro hemoglobínico (Kuhn et al., 1968). Foi demonstrado que a ingestão de ácido ascórbico junto à refeição pode aumentar a absorção e biodisponibilidade do ferro-não-hemínico de 2,2 a 4 vezes (Derman et al., 1980; Hallberg \& Rossander 1984; Monssen 1988).

Os sinais e sintomas da anemia ferropriva são pouco específicos e incluem a anorexia, atonia muscular, fraqueza, tontura, irritabilidade, fadiga, palidez, descoloração das mucosas, apetite pervertido e podem ser identificados na anamnese. No entanto o diagnóstico deve ser confirmado por exame hematológico, uma vez que a anemia ferropriva se caracteriza pela diminuição dos níveis sangüíneos de hemoglobina $(\mathrm{Hb})$, redução do volume de massa eritrocítica e presença de eritrócitos microcíticos e hipocrômicos, o que a diferencia de outros tipos de anemia (Moura et al., 1990).

Existem diversos estudos sobre a prevalência de baixos níveis de hemoglobina, indicativos de anemia ferropriva, em crianças pré-escolares. Serão citados somente os levantamentos mais recentes, realizados na Região Nordeste, em amostras de base populacional: em 1992 foram dois os estudos em amostras representativas do estado da Paraíba e do estado do Piauí, tendo sido encontradas prevalências de 35,6\% (Cariri Benigna et al., 1992) e 33,8\% (Unicef, 1992) respectivamente. Uma amostra de base populacional foi estudada no município de Salvador em 1996, sendo encontradas 46,3\% de crianças com $\mathrm{Hb}$ inferior a 11,0 g/dL (Assis et al., 2000). Pesquisas estaduais de saúde e nutrição foram realizadas em Pernambuco em 1997 e Sergipe em 1998, registrando prevalências de $46,7 \%$ e $31,6 \%$, respectivamente, de crianças de zero a 5 anos com $\mathrm{Hb}$ inferior a 11,0 g/dL (Instituto Nacional..., 1998; Martins et al., 1999).

As manifestações clínicas iniciais da hipovitaminose C são a fadiga, perda de apetite, sonolência, palidez, falta de energia nos membros e articulações, irritabilidade, defeitos dentários, cicatrização lenta de pequenos ferimentos e presença de pequenas hemorragias na pele (Anderson et al., 1988). Já a deficiência grave de ácido ascórbico leva ao escorbuto; em geral a doença se torna sintomática quando os níveis séricos se apresentam abaixo de $0,20 \mathrm{mg} / \mathrm{dL}$. Os sinais e sintomas clássicos desta deficiência grave incluem hemorragias, gengivas edemaciadas e inflamadas, levando à perda de dentes; feridas que não cicatrizam devido à diminuição na síntese do colágeno e infecções secundárias que se desenvolvem facilmente nas áreas sangrentas; distúrbios neuróticos, consistindo de hipocondríase; histeria e depressão seguidos por déficit do desempenho psicomotor são comuns (Mahan \& Arlin, 1995). Alguns autores relataram achados psicométricos desfavoráveis em grupos com carências crônicas de vitaminas, entre as quais a vitamina $\mathrm{C}$; $\mathrm{a}$ suplementação com este nutriente propiciou uma melhora nas funções cognitivas e comportamentais (Heseker et al., 1995).

O Brasil, apesar de ser rico em frutas e verduras, caracteriza-se por grandes desigualdades sociais e econômicas, o que poderia explicar a ocorrência da deficiência da vitamina $C$. Os estudos realizados sobre esta deficiência em nosso meio, se referem à carência vitamínica grave, em ambiente hospitalar (Leão 1977; Amoedo et al., 1985; Santos et al., 1989; Gomes et al., 1993; Oliveira et al., 1994). A maioria dos casos é observado dos 6 aos 24 meses e somente 4\% dos casos incidem em crianças maiores de 2 anos de idade. No Hospital Municipal Jesus, no Rio de Janeiro, foram relatados 13 casos de escorbuto no período de 1973 a 1983, em pacientes com idades de 8 a 36 meses, todos confirmados radiologicamente (Amoedo et al., 1985). No Serviço de Pediatria do Hospital Universitário da Universidade Federal da Paraíba (UFPB), em 1989, Santos e colaboradores acompanharam dois casos de escorbuto infantil no período de 1 ano e 4 meses, demonstrando que com a administração de doses elevadas de ácido ascórbico durante 35 dias, a recuperação ocorreu rapidamente já nas primeiras semanas de tratamento. Gomes et al. (1993) acompanharam em um Hospital de Juiz de Fora, 5 crianças de 6 a 48 meses de idade com sintomatologia clássica de escorbuto. O diagnóstico foi comprovado através de prova terapêutica 
com a vitamina $C$, demonstrando evolução favorável do quadro, para o qual os autores prescreveram uma alimentação equilibrada, fornecendo vitamina $\mathrm{C}$ em doses de 300 a $500 \mathrm{mg} /$ dia durante 4 semanas.

No Nordeste, estudo populacional piloto realizado em crianças escolares de João Pessoa, PB (Costa et al., 1993), revelou que mais de um terço da amostra estudada $(37,0 \%)$ apresentava baixos níveis séricos de vitamina $C$ no sangue $(<0,80 \mathrm{mg} / \mathrm{dL})$. Outros autores estudaram o efeito do estresse físico sobre os níveis séricos de Vitamina $C$ em indivíduos sadios, encontrando níveis significativamente mais elevados após o exercício físico extenuante; assim concluíram que independentemente do estado dietético e fisiopatológico, outros fatores, como o estresse físico, influenciam os níveis séricos de acido ascórbico (Tsuji et al., 1993).

A acerola (Malpighia glabra L.) é uma fruta especialmente rica em vitamina $C$, além de ser também fonte de pró-vitamina A, ferro e cálcio. Pode ser ingerida na forma natural, mas também em preparações como sucos, compotas, geleias, doces, entre outras. Alguns autores determinaram o conteúdo de vitamina $C$ na acerola produzida no Brasil, tendo sido encontrados teores variando de 1040 mg/100g a 1790 mg/100g de parte comestível (respectivamente Leme, 1973 e Visentainer et al., 1997). Também foi estudada a estabilidade da vitamina $C$ do fruto submetido ao processamento e estocagem (Visentainer et al., 1998). O conteúdo de beta caroteno (pró-vitamina A) encontrado na acerola fresca foi de $0,59 \mathrm{mg} / 100 \mathrm{~g}$ (Leme, 1973). A viabilidade do uso de acerola neste estudo baseou-se no fato de que o Nordeste brasileiro é um dos maiores produtores e exportadores do fruto no país, especialmente os estados da Bahia, Paraíba, Pernambuco e Rio Grande do Norte, que dispõem de condições climáticas ideais para o desenvolvimento da aceroleira, que se adapta muito bem ao clima tropical e subtropical (Viglio, 1993).

Este estudo de intervenção foi planejado para analisar o efeito da introdução de um alimento local rico em ácido ascórbico às refeições oferecidas a crianças de 2 a 6 anos matriculadas em creches municipais de João Pessoa. Inicialmente foi determinada a prevalência da deficiência de vitamina $C$ nas crianças estudadas e em seguida, nas crianças com baixos níveis séricos desta vitamina, foi avaliado o efeito da suplementação alimentar com o suco de acerola nos níveis séricos de vitamina $\mathrm{C}$ e na concentração sangüínea de hemoglobina.

\section{CASUÍSTICA E MÉTODOS}

A população estudada foi constituída de 104 crianças de 2 a 6 anos e idade, de ambos os sexos, matriculadas nas seguintes Creches Municipais (e bairros) de João Pessoa: Creche Roberto Vieira Batista - Cristo; Creche Maria José de Miranda Burity - Ilha do Bispo; Creche Vera Lúcia Santana Vieira - Mangabeira; Creche
Margot Trindade - Alto do Mateus; Creche Casulo Arruda Câmara - Bica, as quais foram selecionadas entre as 18 creches municipais existentes.

O protocolo do estudo foi previamente aprovado pelo Comitê de Ética em Pesquisa (CONEP) do Hospital Universitário Lauro Wanderley da UFPB, João Pessoa, PB. Para proceder à coleta de dados cada creche foi visitada com a finalidade de apresentar os objetivos e metodologia do projeto e estabelecer contato entre os pesquisadores, os diretores da instituição e os pais das crianças, aos quais foi solicitado o consentimento para a participação de seus filhos no estudo. Das crianças inicialmente recrutadas para o estudo, cinco apresentaram níveis séricos de vitamina C inferiores a $0,20 \mathrm{mg} / \mathrm{dL}$ indicativos de deficiência vitamínica grave; estas foram submetidas a um exame clínico-nutricional padronizado, tratadas com vitamina C medicamentosa e excluídas do estudo. Também por questões éticas, não foi incluído no desenho deste estudo um grupo controle (placebo) com crianças deficientes em vitamina $\mathrm{C}$.

Os dados foram coletados por uma equipe devidamente treinada, composta por 3 nutricionistas, uma médica, uma enfermeira, uma bioquímica, um assistente social e três alunos de graduação da UFPB (Nutrição e Medicina), bolsistas do PIBIC. O sangue (cerca de $4 \mathrm{~mL}$ ) foi colhido por venipuntura pela bioquímica da equipe, com as crianças em jejum, empregando seringas e agulhas BD descartáveis. A alíquota do sangue destinada à dosagem de hemoglobina foi adicionada de EDTA sódico, enquanto que a porção restante foi acondicionada em tubo de ensaio seco, para a coagulação e obtenção do soro. O material foi imediatamente transportado em condições apropriadas para o Laboratório de Nutrição e Saúde Pública do Departamento de Nutrição da UFPB, onde foi analisado.

A determinação do estado vitamínico $C$ sérico foi realizada por dosagem direta, pelo método fotométrico de Lowry et al. (1945) modificado por Costa (1992). A interpretação dos níveis de ácido ascórbico foi a seguinte: acima de $0,80 \mathrm{mg} / \mathrm{dL}$ - níveis normais; de 0,20 até $0,80 \mathrm{mg} / \mathrm{dL}$ - deficiência moderada e menor de $0,20 \mathrm{mg} / 100 \mathrm{~mL}$ - deficiência grave (Nutrition Canadá, 1973).

A dosagem de hemoglobina foi realizada utilizando-se o método da Cianometahemoglobina com leitura em espectrofotômetro a $540 \mathrm{~nm}$; crianças de 2 a 6 anos de idade, devem ser consideradas anêmicas quando os níveis de hemoglobina são inferiores a 11,0 g/dL (Organización Mundial..., 1990; Demaeyer, 1991), sendo que níveis abaixo de 9,0 g/dL caracterizam a anemia grave. Para a dosagem de hemoglobina houve uma perda amostral de 20 crianças, causada por problemas técnicos laboratoriais em uma das creches, resultando então em um total de 84 crianças com esta determinação.

Foi realizada a avaliação nutricional utilizando-se o índice antropométrico Peso/Idade, tendo como parâmetro de avaliação o Desvio-Padrão escore (DP) da população de referência da Organización Mundial... (1983). A idade 
foi calculada a partir da data de nascimento obtida na ficha de inscrição. Foram seguidos os procedimentos padronizados (Barros \& Victora, 1991) sendo o peso obtido com as crianças em jejum e com o mínimo de vestimenta, em balança da marca Filizola para até 150 kg, com divisões de $100 \mathrm{~g}$.

Para a suplementação alimentar das crianças com baixos níveis de vitamina $C$ sérica $(0,20-0,80 \mathrm{mg} / \mathrm{dL})$, foram oferecidos $150 \mathrm{~mL}$ de suco de acerola com uma quantidade média de 565 mg de ácido ascórbico/100mL de suco. A suplementação foi realizada durante o almoço, por um período de 35 dias consecutivos. O suco foi preparado a partir da polpa processada em laboratório: a quantidade exata de polpa suficiente para preparar o suco era retirada do congelador e colocada em recipiente fechado para descongelar, durante 20 a 30 minutos, sendo em seguida homogeneizados com auxílio de uma colher até a dissolução total da polpa e do açúcar (adicionado em concentração de cerca de 10\%). A aceitação do suco foi excelente, não tendo sido registrado nenhum caso de recusa.

Para o tratamento estatístico dos dados, utilizou-se o programa SPSS for WINDOWS, Versão 5.1 na determinação de freqüências percentuais, médias, desvios-padrão e aplicação dos testes "t", Qui-Quadrado $\left(X^{2}\right)$ de Pearson e " $F$ " na Análise de Variância (ANOVA) (Norusis, 1992). O intervalo de confiança empregado foi de $95 \%$.

\section{RESULTADOS}

A distribuição das crianças segundo os níveis séricos de vitamina $C$, mostrou uma alta prevalência de níveis deficientes $(69,2 \%)$, que se distribuiu uniformemente segundo o sexo $(p=0,947)$. Este resultado serviu de base para identificar o sub-grupo de 72 crianças com baixos níveis de Vitamina $C$, que recebeu a suplementação com acerola. Não foram registrados sinais e ou sintomas de escorbuto, apesar da alta prevalência de crianças com níveis séricos deficientes (Tabela 1).

Nas 97 crianças com antropometria completa, foi analisada a relação entre o indicador Peso/Idade (abaixo de -2,0 DP) e os níveis de vitamina C. De 30 crianças com níveis normais de vitamina C, $21(70,0 \%)$ apresentaram peso normal para a idade e $9(30,0 \%)$ estavam com baixo peso. No grupo de 67 crianças com baixos níveis de vitamina C, 43 (64,2\%) eram normais, 23 (34,3\%) com baixo peso e uma $(1,5 \%)$ apresentou sobrepeso. $\mathrm{Na}$ população estudada verificou-se que não houve dependência entre o estado nutricional (peso/idade) e os níveis de vitamina $C(p=0,7146)$.

A prevalência de anemia foi de $22,6 \%$ no sub-grupo com níveis iniciais normais de vitamina $\mathrm{C}$, enquanto que nas crianças deficientes nesta vitamina, foi de 43,3\%. Pela aplicação do teste $X^{2}$ de Pearson verificou-se que as variáveis presença de anemia e baixos níveis séricos de vitamina $C$, mostraram uma relação limítrofe em termos de significância $(p=0,054)$ (Tabela 2).

Por conta desta possível associação, decidiu-se analisar a correlação entre estas duas variáveis, que são contínuas (Figura 1); pode-se notar que, apesar da evidente dispersão, havia uma tendência no sentido que níveis adequados de vitamina $\mathrm{C}$ (superiores a $0,80 \mathrm{mg} / \mathrm{dL}$ ) estarem associados a níveis adequados de hemoglobina (superiores a $11,0 \mathrm{~g} / \mathrm{dL})$; o teste " $\mathrm{F}$ " indicou uma correlação estatisticamente significativa $(p=0,01)$.

Observa-se que a concentração média de vitamina C no grupo de 72 crianças com carência desta vitamina (níveis de 0,20 a $0,80 \mathrm{mg} / \mathrm{dL}$ ) na linha base, oscilou entre $0,40+0,19 \mathrm{~m} / \mathrm{dL}$ na Creche II e 0,67 + 0,12 mg/dL na Creche IV. Pode-se observar que após a suplementação os níveis médios de Vitamina $C$ no soro apresentaram-se normais em todas as 5 Creches, oscilando entre 1,09+ 0,27 na Creche IV e 1,74 + 0,44 na Creche III. Com relação aos valores médios das concentrações de Vitamina $C$ no plasma em toda a sub-amostra que recebeu suplementação $(n=72)$ verifica-se que a média final de $1,43+0,43$ apresentou-se estatisticamente superior $(p<0,001)$ ao valor encontrados antes da suplementação, que foi de 0,52 + 0,20 de acordo com o Teste " $\mathrm{t}$ " para amostras pareadas. Foi comparado a situação em relação à média do teor de hemoglobina, que apresentou um aumento significativo de 11,17 + 1,46 g/dL antes, para $12,22+1,52 \mathrm{~g} / \mathrm{dL}$, após a suplementação $(\mathrm{p}<0,001 \mathrm{de}$ acordo com o teste " $\mathrm{t}$ " para amostras pareadas) (Tabela 3 ).

Tabela 1. Prevalência de carência de vitamina $C$ em crianças de 2 a 6 anos em creches municipais de João Pessoa, Paraíba, segundo o sexo.

\begin{tabular}{|c|c|c|c|c|c|c|}
\hline \multirow{3}{*}{ Sexo } & \multicolumn{4}{|c|}{ Concentração sérica de vitamina C (mg/dL) } & \multirow{2}{*}{\multicolumn{2}{|c|}{ Total }} \\
\hline & \multicolumn{2}{|c|}{ Normal $(>0,80)$} & \multicolumn{2}{|c|}{ Baixo $(0,20-0,80)$} & & \\
\hline & $\mathrm{n}$ & $\%$ & $\mathrm{n}$ & $\%$ & $\mathrm{n}$ & $\%$ \\
\hline Masculino & 18 & 31,0 & 40 & 69,0 & 58 & 100,0 \\
\hline Feminino & 14 & 30,4 & 32 & 69,6 & 46 & 100,0 \\
\hline Total & 32 & 30,8 & 72 & 69,2 & 104 & 100,0 \\
\hline
\end{tabular}

$\mathrm{p}>0,05$ ( $X^{2}$ Pearson). 
Tabela 2. Prevalência da anemia em crianças de 2 a 6 anos em creches municipais de João Pessoa, Paraíba, segundo a concentração sérica de vitamina C.

\begin{tabular}{|c|c|c|c|c|c|c|}
\hline \multirow{3}{*}{$\begin{array}{l}\text { Classificação dos níveis de } \\
\text { Hemoglobina }\end{array}$} & \multicolumn{4}{|c|}{ Concentração sérica de vitamina $\mathrm{C}(\mathrm{mg} / \mathrm{dL})$} & \multirow{2}{*}{\multicolumn{2}{|c|}{ Total }} \\
\hline & \multicolumn{2}{|c|}{ Normal $(>0,80)$} & \multicolumn{2}{|c|}{ Baixo $(0,20-0,80)$} & & \\
\hline & $\mathrm{n}$ & $\%$ & $\mathrm{n}$ & $\%$ & $\mathrm{n}$ & $\%$ \\
\hline Normal $\mathrm{Hb} \geq 11 \mathrm{~g} / \mathrm{dL}$ & 24 & 77,4 & 30 & 56,6 & 54 & 64,3 \\
\hline Anemia $\mathrm{Hb}<11 \mathrm{~g} / \mathrm{dL}$ & 7 & 22,6 & 23 & 43,3 & 30 & 35,7 \\
\hline Total & 31 & 100,0 & 53 & 100,0 & 84 & 100,0 \\
\hline
\end{tabular}

$\mathrm{p}>0,054$ ( $\mathrm{X}^{2}$ Pearson).

Tabela 3. Níveis médios de vitamina $C$ e de hemoglobina em crianças de 2 a 6 anos em creches municipais de João Pessoa, Paraíba, antes e após a suplementação com suco de acerola (Malpighia glabra L.) por 35 dias, segundo a creche.

\begin{tabular}{|c|c|c|c|c|}
\hline \multirow[t]{2}{*}{ Creche } & \multicolumn{2}{|c|}{$\begin{array}{c}\text { Vitamina C sérica }(\mathrm{mg} / \mathrm{dL}) \\
\text { (Média + desvio-padrão) }(\mathrm{n}=72)\end{array}$} & \multicolumn{2}{|c|}{$\begin{array}{c}\text { Teor de Hemoglobina }(\mathrm{g} / \mathrm{dL}) \\
\text { (Média + desvio-padrão) }(\mathrm{n}=54)\end{array}$} \\
\hline & Antes & Depois & Antes & Depois \\
\hline Creche I $\quad(n=18)$ & $0,48 \pm 0,19$ & $1,45 \pm 0,53$ & n.d. & n.d. \\
\hline Creche II $\quad(n=23)$ & $0,40 \pm 0,19$ & $1,40 \pm 0,27$ & $10,70 \pm 1,70$ & $11,02 \pm 0,80$ \\
\hline Creche III $(n=11)$ & $0,57 \pm 0,16$ & $1,74 \pm 0,44$ & $12,45 \pm 1,12$ & $12,35 \pm 1,60$ \\
\hline Creche IV $(n=10)$ & $0,67 \pm 0,12$ & $1,09 \pm 0,27$ & $11,14 \pm 0,89$ & $13,71 \pm 0,84$ \\
\hline Creche V $(n=10)$ & $0,67 \pm 0,08$ & $1,48 \pm 0,44$ & $10,85 \pm 0,85$ & $13,22 \pm 1,11$ \\
\hline Total & $0,52 \pm 0,20^{*}$ & $1,43 \pm 0,43^{*}$ & $11,17 \pm 1,46^{*}$ & $12,22 \pm 1,52^{*}$ \\
\hline
\end{tabular}

$* \mathrm{p}<0,001$ (Teste “" $\mathrm{t}$ ); n.d. = não determinado.

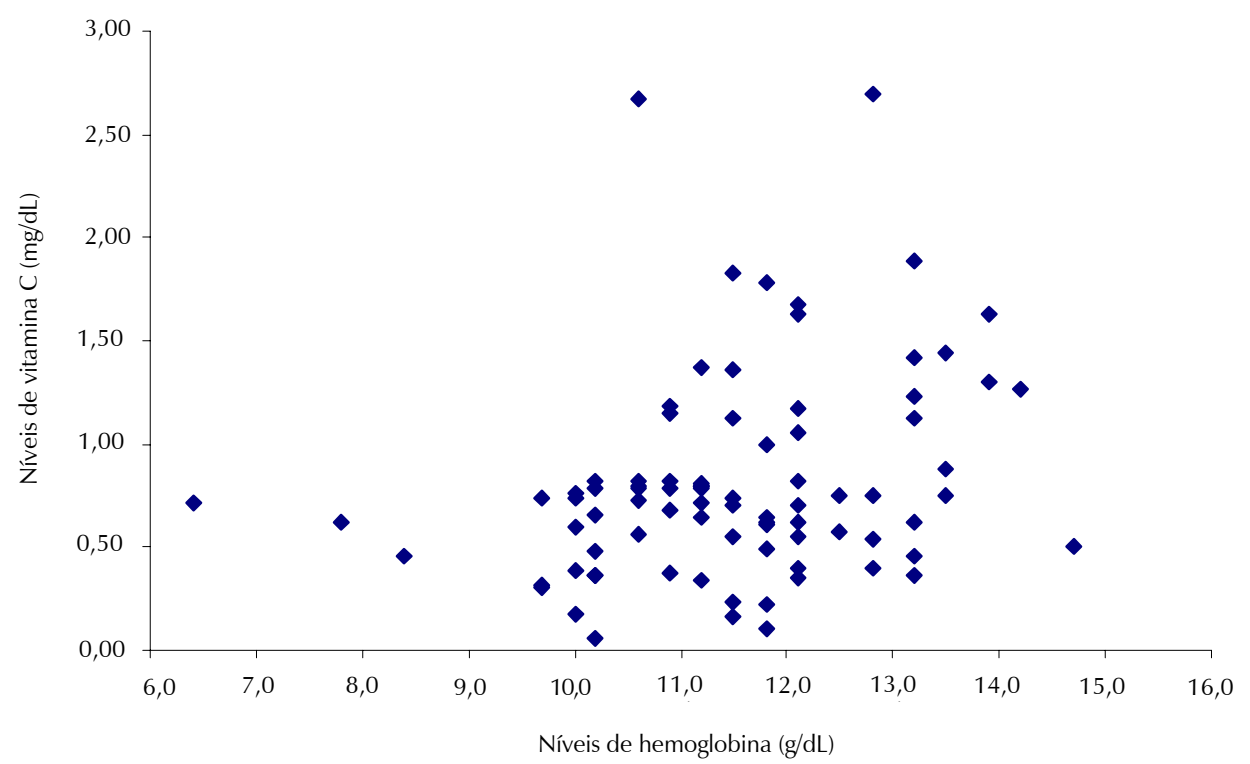

Figura 1. Correlação entre os níveis sangüíneos iniciais de vitamina C e de hemoglobina em crianças de 2 a 6 anos de creches municipais de João Pessoa, Paraíba. 
Após a suplementação 67 das 72 crianças, ou seja $93 \%$, apresentavam nível sérico de vitamina $C$ na faixa da normalidade $(>0,80 \mathrm{mg} / \mathrm{dL})$.

\section{DISCUSSÃO}

O nível de ácido ascórbico nos leucócitos têm sido citado freqüentemente como um bom indicador do estado vitamínico $\mathrm{C}$ em humanos, porém o plasma e o soro são também usados por muitos para verificar as reservas orgânicas de vitaminas e para determinar o estado nutricional de ácido ascórbico (Lee et al., 1988; Omaye et al., 1988).

Estudo realizado por Blanchard (1991) com mulheres e homens jovens e idosos mostra o perfil de depleção/repleção da vitamina $\mathrm{C}$ através da determinação desta vitamina no plasma. A concentração de ácido ascórbico no plasma ou soro, está geralmente correlacionado com a ingestão alimentar de vitamina $\mathrm{C}$ e com a concentração de ácido ascórbico nos leucócitos (Guilland \& Lequeu, 1995). Estudos de depleção e repleção sugerem que a "ascorbemia" é mais sensível ao aporte alimentar recente, enquanto que as taxas intra-leucocitárias são reflexos dos estoques celulares e do pool total do organismo (Jacob et al., 1987; Sauberlich et al., 1989).

Uma revisão de literatura indica que populações que consomem a longo prazo níveis de vitamina $\mathrm{C}$ maiores que o da Recommend Dietary Allowances (RDA) ( $\geq 60 \mathrm{mg} /$ dia) na forma de alimentos e/ou suplementos, têm reduzido os riscos de câncer, doenças cardiovasculares e catarata (Bendich 1993). Contudo megadoses desta vitamina não são inócuas: segundo Johnston \& Yen (1994), as doses de ácido ascórbico acima de $1 \mathrm{~g}$ por dia estimulam a hiperglicemia pós-prandial em diabéticos, como interferem com a vitamina $B_{12}$ nos alimentos, no organismo humano, ou em ambos, sendo que indivíduos que ingerem megadoses desta vitamina podem desenvolver enfermidades por deficiência de $\mathrm{B}_{12}$ (Organización Panamericana..., 1991). Estudando a tolerância e o efeito de altas doses de ácido ascórbico Hanck (1982) concluiu que estas podem ser prejudiciais quando administradas por um longo período sem supervisão médica, podendo desencadear diversas doenças, além das citadas anteriormente. Por sua vez, doses elevadas de ácido ascórbico, por períodos prolongados, podem levar, quando suspensas, ao desencadeamento de um quadro de escorbuto (Rhead et al., 1971).

Para os indicadores da anemia, existe consenso de que os níveis sangüíneos de hemoglobina são úteis no diagnóstico da doença (Organización Mundial..., 1990; DeMaeyer et al., 1991), mas que os mesmos não diferenciam o tipo e/ou a etiologia da anemia. A anemia mais comum em nosso meio é, a ferropriva; durante a infância, há períodos rápidos de crescimento nos quais as necessidades aumentadas de ferro nem sempre são compensados pela ingestão alimentar, sendo os principais determinantes da anemia ferropriva a dieta deficiente em ferro, como também pobre em fatores promotores da absorção do ferro, como a vitamina C (Kuhn et al., 1968; Derman et al., 1980; Hallberg \& Rossander 1984; Monssen, 1988). Contudo anemias mais raras como as hereditárias, não podem ser descartadas; o diagnóstico diferencial exigiria um hemograma completo e eletroforese, exames nem sempre possíveis de ser realizados em condições de trabalho de campo.

Diversos autores estudaram o efeito da suplementação da vitamina $\mathrm{C}$ no estado hematológico em seres humanos (Pureur, 1984; Seshadri et al., 1985; Ajayi et al., 1990; Cesar, 1990; Mao \& Yao, 1992; Sharma \& Mathur, 1995). No primeiro estudo em crianças com deficiência de ferro, mostrou a eficácia da suplementação da vitamina $\mathrm{C}$ com o controle da ingestão de ferro, em seus pacientes. Seshadri et al. (1985) suplementaram crianças anêmicas vegetarianas com vitamina C por 60 dias e demonstraram aumento significativo nos níveis de hemoglobina. Em adultos a suplementação com vitamina $\mathrm{C}$ e riboflavina produziu um aumento significante na hemoglobina, hematócrito e contagem de hemácias (Ajayi et al., 1990). Estudando 380 crianças escolares Cesar (1990) testou a efetividade da intervenção com suco contendo vitamina $\mathrm{C}$ oferecido diariamente por quatro meses junto à merenda escolar e obteve como resultado uma diminuição percentual de $20 \%$ na prevalência de anemia nos grupos controle experimental, resultado que, segundo o autor, sugeriu a não eficácia da intervenção. Mao e Yao (1992) estudou o efeito dose-resposta da suplementação com vitamina $C$ por 8 semanas em crianças anêmicas e concluíram que a suplementação com ácido ascórbico, por si só, pode controlar a anemia ferropriva e que a dosagem de $50 \mathrm{mg} / \mathrm{dia}$ foi a mais efetiva. Sharma \& Mathur (1995) ofereceram um suplemento de vitamina C a um grupo de vegetarianos, registrando aumentos (significativos) de 8\% na média de hemoglobina, de 17\% no ferro sérico e de $23 \%$ na saturação de transferrina; os autores sugeriram que a suplementação com vitamina $C$ pode ser mais eficaz do que a suplementação com ferro no controle da anemia ferropriva.

Os resultados aqui obtidos apontam na mesma direção, pois embora com uma amostra reduzida, foi possível observar uma correlação entre os níveis inicias de vitamina $C$ e hemoglobina e, além disto, um aumento significativo nos níveis médios de vitamina $C$ e de hemoglobina após 35 dias de suplementação com acerola. Tendo em vista a disponibilidade e o baixo custo deste fruto na região é aconselhável a inclusão em programas de alimentação para populações de alto risco para a anemia, exemplo do Programa de Alimentação Escolar, Programa de Combate às Carências Nutricionais e outros.

\section{REFERÊNCIAS BIBLIOGRÁFICAS}

AJAYI, O.A, OKIKE, O.C., YUSUF, Y. Haematological response to supplements of riboflavin and ascorbic acid in Nigerian young 
adults. European Journal of Haematology, Copenhagen, v.44, n.4, p.209-212, 1990.

AMOEDO, A.R., PINHEIRO, P.C.M.S., MARTIRE, T. Escorbuto: aspectos clínicos e radiológicos do escorbuto, Hospital Municipal Jesus (Rio de Janeiro) 1973-83: análise de 13 casos. Journal of Pediatrics, St. Louis, v.58, n.4, p.157-192, 1985.

ANDERSON, L. et al. The effects os increasing weekly doses of ascorbate on certain cellular and humoral immune functions in volunteers. American Journal of Clinical Nutrition, Bethesda, v.33, n.6, p.71-76, 1988.

ASSIS, A.M.O., BARRETO, M.L., SANTOS, L.M.P., SAMPAIO, L.R., MAGALHÃES, L.P., PRADO, M.S., SANTOS, N.S., GALVÃO, N.M.S., SILVA, R.C.R., OLIVEIRA, V.A. Condições de vida, saúde e nutrição na infância em Salvador. Salvador : Artes Gráficas, 2000. 163p.

BARROS, F.C., VICTORA, C.G. Epidemiologia da saúde infantil: um manual para diagnósticos comunitários. São Paulo : Hucitec, 1991. Cap.6: Exame físico e antropométrico. p.89105.

BENDICH, A. Physiological role of antioxidants in the immune system. Journal of Dairy Science, Champaign IL, v.76, n.9, p.2789-2794, 1993.

BLANCHARD, J. Vitamins: depletion and repletion kinetics of vitamin $C$ in humans. [s.l.] : American Institute of Nutrition, 1991. p.170-176.

CARIRI BENIGNA, M.J., OLIVEIRA, R.S., LOLA, M.F., ASSIS, A.S., RIVERA, M.A.A. MIRANDA, S.S., ASCIUTTI MOURA, L.S. Anemia nutricional no Estado da Paraíba. In: CONGRESSO BRASILEIRO DE NUTRIÇÃO, 3., 1992, Rio de Janeiro. Resumos. Rio de Janeiro : CONBRAN, 1992. p.50.

CESAR, A.T. O uso de ácido ascórbico no controle da deficiência de ferro utilizando a estrutura do Programa de Merenda Escolar. São Paulo, 1990. p.76. Dissertação (Mestrado) - Universidade de São Paulo, 1990.

COSTA, M.J.C. Statut vitaminique biochimique. Nancy, France : INSERM-CNRS, 1992. (Rapport Techinique de Post-Doctoral - Université de Nancy, Center de Médicine Preventive).

COSTA, M.J.C., RIVERA, M.A.A., CARIRI BENIGNA, M.J., LINS, M.E.P., ARAÚJO, L.S.A., SOUZA, R.G. Avaliação do estado nutricional vitamínico $\mathrm{C}$ em escolares: estudo piloto. Revista de Ciência, Cultura e Saúde, João Pessoa, v.12, n.8, p.85-91, 1993.

DEMAEYER, E.M. Prévenir et combattre l' anémie ferriprive dans le cadre des soins de santé primaires: guide à I' usage des administrateurs de la santé et des responsables de programme. Geneve: OMS, 1991. p.61.

DERMAN, D.P., BOTHWELL, T.H., MACPHAIL, A.P., TORRANCE, J.D., BEZWODA, W.R., CHARLTON, R.W., MAYET, F.G. Importance of ascorbic acid in the absortion of iron from infant foods. Scand J Haematol, v.25, n.3, p.193-201, 1980.

GOMES, J.M., GORESKE, C.M., CASTRO, F.A., AMIN, A.B. Estudo do escorbuto no Serviço de Pediatria do Hospital Universitário da Universidade Federal de Juiz de Fora. Revista Médica de Minas Gerais, v.3, n.1, p.16-18, 1993.

GUILLAND, J.C., LEQUEU, B. As vitaminas: do nutriente ao medicamento. São Paulo : Santos, 1995. p.357.

HANCK, A. Tolerance and effects of high doses of ascorbic acid. International Journal for Vitamin and Nutrition Research, Bern, v.23, p.221-238, 1982. Supplement.
HALLBERG, L., ROSSANDER, L. Improvement of nutrition in developing countries: comparison of adding meat, soy protein, ascorbic acid, citric acid and ferrous sulphate on iron absorption from a simple Latin American-type meal. American Journal of Clinical Nutrition, Bethesda, v.39, n.4, p.577-583, 1984.

HESEKER, H., KUBLER, W., WESTENHOFER, J. Interactions of vitamins with mental performance. Bibliotheca Nutritioet Dieta, Basel, v.52, p.43-55, 1995.

INSTITUTO NACIONAL DE NUTRIÇÃO. II Pesquisa Estadual de Saúde e Nutrição. Recife, 1998. 117p.

JACOB, R.A., SKALA, J.H., OMAYE, S.T. Biochemical indices of human vitamin $C$ status. American Journal of Clinical Nutrition, Bethesda, v.60, n.5, p.818-826, 1987.

JOHNSTON, C.S., YEN, M.F. Megadose os vitamin C delays insulin response to a glicose challenge in normoglicemic adults. American Journal of Clinical Nutrition, Bethesda, v.60, p.735-738. 1994.

KHUN, I.N., LAYRISSE, M., ROCHE, M., MARTINEZ, C. Observations on the mechanism of iron absortion. American Journal of Clinical Nutrition, Bethesda, v.21, n.10, p.1184-1188, 1968.

LEÃO, E. Contribuição ao estudo do escorbuto na criança. Belo Horizonte, 1977. 141p. Dissertação (Mestrado) - Faculdade de Medicina, Universidade Federal da Minas Gerais, 1977.

LEE, W., DAVIS, K.A., RETTMER, R.L., LABBE, R.F. Ascorbic acid status: biochemical and clinical considerations. American Journal of Clinical Nutrition, Bethesda, v.48, n.2, p.286-290, 1988.

LEME JR, J., FONSECA, H., NOGUEIRA, J.N. Variação do teor de ácido ascórbico e beta-caroteno em cereja das Antilhas (Malpighia p.L) liofilizada. Archivos Latinoamericanos de Nutrición, Guatemala, v.23, n.2, p.207-215, 1973.

LOWRY, O.H., LOPEZ, J.A., BESSEY, O.A. The determination of ascorbic acid in small amounts of blood serum. Journal of Biological Chemistry, Baltimore, v.160, p.609-615, 1945.

MAHAN, L.K., ARLIN, M.T. Krause: alimentos, nutrição e dietoterapia. São Paulo : Rocca, 1995. p.957.

MAO, X., YAO, G. Effect of vitamin $\mathrm{C}$ supplementations on iron deficiency anemia in Chinese children. Biomedical and Environmental Sciencies, San Diego CA, v.5, n.2, p.125-129, 1992.

MARTINS, M.C., SANTOS, L.M.P., LIMA, A.P., ASSIS, A.M.O. Perfil nutricional: desnutrição e anemia em crianças no estado de Sergipe. In: CONGRESSO NACIONAL DA SOCIEDADE BRASILEIRA DE ALIMENTAÇÃO E NUTRIÇÃO, 5., 1999, São Paulo. Anais... São Paulo: SBAN, 1999. p.184.

MONSSEN, E.R. Iron nutrition and absorption: dietary factors which impact iron availability. Journal of the American Dietetic Association, Chicago, v.88, n.7, p.786-790, 1988.

MOURA, E.C. et al., Caracterização da anemia ferropriva numa Unidade de Atenção Primária a Saúde. Revista de Nutrição da PUCCAMP, Campinas, v.3, n.1, p.9-20, 1990.

NORUSIS, M.J. SPSS for Windows: base system user guide. Release 5.0. Chicago : SPSS 1992. p.645.

NUTRITION CANADÁ. Interpretative Standards. Ottawa : Information Canada, 1973. p.46. (National Survey).

OLIVEIRA, G.F., PINHO, M.R.X., MOURA, E.F.A. Escorbuto (apresentação de caso). Arquivos Brasileiros de Pediatria, Rio de Janeiro, v.1, n.4, p.97-100, 1994. 
OMAYE, S.T., SKALA, J.H., JACOB, R.A. Rebound effect with ascorbic acid in adults males (Letter). American Journal of Clinical Nutrition, Bethesda, v.48, n.2, p.379-390, 1988.

ORGANIZACIÓN MUNDIAL DE LA SALUD. Medición del cambio estado nutricional. Ginebra, 1983. 106p.

ORGANIZACIÓN MUNDIAL DE LA SALUD. Primer informe sobre la situación de la nutrición en el mundo. Washington DC, 1990. p.30-32. (Cuaderno Técnico, 28).

ORGANIZACIÓN PANAMERICANA DE LA SALUD. Conocimientos actuales sobre nutrición. 6.ed. Washington DC : ILSI, 1991. p.152-162.

PUREUR, J.L. Nutritional iron deficiency: is there a connection, in some infants, with subclinical vitamin $\mathrm{C}$ deficiency? Annales de Pédiatrie, Paris, v.31, n.11, p.961-964, 1984.

RHEAD, W.J., SCHAUZER, G.N. Risks of long-term ascorbic acid over dosage. Nutrition Reviews, New York, v.29, n.11, p.262-263, 1971.

SANTOS, E.P. et al. Escorbuto infantil: relato de dois casos e revisão de literatura. Revista de Ciência, Cultura e Saúde, João Pessoa, v.11, n.2, p.127-146, 1989.

SAUBERLICH, H.E., KRETSCH, M.J., TAYLOR, P.C., JOHNSON, H.L., SKALA, J.H. Ascorbic acid and erythorbic acid metabolism in nonpregnant women. American Journal of Clinical Nutrition, Bethesda, v.50, n.5, p.1039-1049, 1989.
SESHADRI, S., SHAH., A., BHADE, S. Haematologic response of anaemic preschool to ascorbic acid supplementation. Human Nutr: Appl Nutr, New Delhi, v.39, n.2, p.151-154, 1985.

SHARMA, D.C., MATHUR, R. Correction of anemia and iron deficiency in vegetarians by administration of ascorbic acid. Indian Journal of Physiology and Pharmacology, New Delhi, v.39, n.4, p.403-406, 1995.

TSUII, H., SEABRA, M.E.G., MATSUBARA, B.B., BURINI, R.C. Efeito de idade, sexo e estresse físico sobre os níveis séricos de vitamina C em indivíduos sadios. Revista Brasileira de Patologia Clínica, Rio de Janeiro, v.29, n.3, p.83-86, 1993.

UNICEF. Crianças e adolescentes no Piauí: saúde, educação e trabalho. Teresina, 1992. 78p. (Governo do Estado do Piauí).

VIGLIO, E.C.B.L. O potencial da acerola. Revista Conjuntura Econômica, Rio de Janeiro, v.47, n.9, p.53-54, 1993.

VISENTAINER, J.V., VIEIRA, O.A., MATSUSHITA, M., SOUZA, N.E. Características físico-químicas da acerola Malpighia g.L. produzida na região de Maringá. Archivos Latinoamericanos de Nutrición, Guatemala, v.47, n.1, p.70-72, 1997.

VISENTAINER, J.V., VIEIRA, O.A., MATSUSHITA, M., SOUZA, N.E. Vitamin $C$ in Barbados cherry Malpighia g.L. pulp submitted to processing and to different forms of storage. Archivos Latinoamericanos de Nutrición, Guatemala, v.48, n.3, p.256-259, 1998.

Recebido para publicação em 14 de julho de 1999 e aceito em 8 de junho de 2000. 